\title{
Is the patient choice the best choice?
}

\author{
Angelo Hooker ${ }^{1}$ \\ ${ }^{1}$ Zaans Medical Center (ZMC)
}

May 17, 2021

\section{Is the patient choice the best choice?}

Angelo B. Hooker, M.D.

Uterine fibroids, the most common type of tumor among women of reproductive age are often associated with symptoms and a reduce quality of life (Stewart et al. BJOG 2017;124:1501-12). Hysterectomy has been traditionally the primary approach for the management of symptomatic fibroids after unsuccessful medical treatment. In symptomatic women who do not want to undergo hysterectomy, myomectomy and uterineartery embolization (UAE) are therapeutic options, but solid evidence on the cost-effectiveness is lacking (Gupta et al. Cochrane Database Syst Rev 2012;5:CD005073).

The FEMME trial, a multicenter randomized controlled trial compared myomectomy with UAE in 254 premenopausal women with symptomatic uterine fibroids wishing to preserve their uterus and fertility (Manyoda et al. N Engl J Med 2020;383:440). Dikshyanta and co-workers conducted an economic evaluation alongside. UAE was dominated by myomectomy. UAE was associated with higher mean costs (£645 and £352 over two and four year horizon) and lower health-related quality-of-life (difference of 0.09 after both two and four years) when compared with myomectomy. Sensitivity analyses did not influenced the results.

Although the authors should be complimented for conducting this economic valuation, the results should be interpreted cautiously. A substantial number of women amendable for the FEMME trail were not recruited due to preference for a particular treatment. The analysis was conducted according to intention-to-treat principle but of the participants randomly assigned only (98/127) $77 \%$ women underwent embolization and (105/127) $83 \%$ underwent myomectomy. Furthermore, $82 \%$ of the myomectomy procedures were open abdominal procedures and only $18 \%$ laparoscopic. Noteworthy, after four years up to $50 \%$ of response of the participants on the health-related quality-of-life questionnaires were missing, a significant percentage.

The cost-effectiveness of different treatment is influenced by different factors, including settings, population, time horizon, costs, perspectives, method of analysis and assumptions made for analysis, making generalizability of the findings and comparison with other studies difficult. Due to restriction of the framework used, it was not possible to take patient preference and possible preferences of women for a less invasive procedure into account. Although AUE was dominated by myomectomy, less costly and provided better health-related quality-of-life scores, the differences after 4 years follow-up were relatively small and both treatments significantly improved health-related quality of life.

Despite the reported limitations, Dikshyanta and co-authors must be highly complimented for conducted this economic evaluation. This study provides valuable information. Clinicians are able to better inform women with symptomatic fibroids wishing to avoid a hysterectomy and to help them in the decision making process, including the costs-effectiveness of the procedures. As women may add value to UAE as a non-surgical procedure, women should have the options to choose between both procedures.

It may be of value to continue to follow-up the participants to establish if the reported differences between the treatment methods still persist after a longer period. Further research on the cost-effectiveness of (new) 
treatment methods, like MRgHIFU for symptomatic uterine fibroids, especially in women wishing to avoid hysterectomy and preserve their fertility, are still required. 